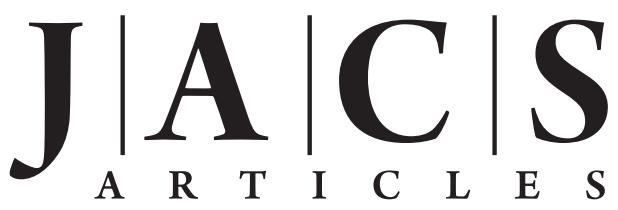

Published on Web 06/28/2010

\title{
Mechanical Distortion of Protein Receptor Decreases the Lifetime of a Receptor-Ligand Bond
}

\author{
Senli Guo, Nan Li, Nimit Lad, Chad Ray, and Boris B. Akhremitchev* \\ Department of Chemistry, Duke University, Durham, North Carolina 27708
}

Received February 9, 2010; E-mail: boris.a@duke.edu

\begin{abstract}
Substantial experimental evidence indicates that the mechanical force applied to pull apart noncovalent molecular bonds (such as receptor-ligand pairs) can significantly decrease the bond lifetime. This evidence is often generated in single-molecule experiments that are designed to specifically test effects of pulling forces. However, the effect of compressive forces on the lifetime of receptor-ligand bonds remains largely unexplored. Here we extend the common usage of the atomic force microscopy technique to study whether compressive forces applied to bound streptavidin-biotin species can significantly accelerate the rate of dissociation. Presented experimental data indicate that compressive forces can substantially decrease the lifetime of the molecular bond. Surprisingly, the efficiency of accelerating dissociation by compressive forces sometimes exceeds the enhancement of the dissociation rate measured in pulling experiments, indicating that compressive forces applied to the bound species might be efficiently used to control the lifetime of adhesion bonds.
\end{abstract}

\section{Introduction}

Mechanical forces play an important role in many fundamental biological processes, such as cell adhesion and motion, the function of motor proteins, and mechanical sensors. ${ }^{1-3}$ When applied to atoms or molecules, mechanical forces can significantly affect the course of chemical reactions and the lifetime of molecular bonds. ${ }^{4,5}$ Kinetic effects of mechanical forces on the dissociation rate of molecular bonds are attributed to tilting of the energy landscape by external force. The rate of escape from a bound state in the tilted potential differs from the rate of escape at zero force. In the past this concept has been applied to explain various phenomena involving mechanical forces: for example, by Eyring in a 1936 article $^{6}$ to develop kinetic theory of viscosity, by Zhurkov in a 1965 article $^{7}$ on the mechanical strength of solids, and by De Gennes in a 1974 article $^{8}$ on forceinduced scission of individual polymer molecules in the shear flow. In application to the receptor-ligand bonds, Bell also used this concept in his 1978 article $^{9}$ to develop a phenomenological model of force resistance of adhesion bonds between cells. In contemporary literature this model is often referred to as the Bell theory. According to this theory the dissociation rate is accelerated by applied force $F$ by a factor of $\exp \left(F x^{\ddagger} /\left(k_{\mathrm{B}} T\right)\right)$, where $x^{\ddagger}$ is a characteristic distance between the bound state and the transition state, $k_{\mathrm{B}}$ is the Boltzmann constant, and $T$ is

(1) Bustamante, C.; Chemla, Y. R.; Forde, N. R.; Izhaky, D. Annu. Rev. Biochem. 2004, 73, 705-748.

(2) Vogel, V.; Sheetz, M. Nat. Rev. Mol. Cell Biol. 2006, 7, 265-275.

(3) Fletcher, D. A.; Geissler, P. L. Annu. Rev. Phys. Chem. 2009, 60, 469-486.

(4) Evans, E. Ann. Rev. Biophys. Biomol. Struct. 2001, 30, 105-128.

(5) Hickenboth, C. R.; Moore, J. S.; White, S. R.; Sottos, N. R.; Baudry, J.; Wilson, S. R. Nature 2007, 446, 423-427.

(6) Eyring, H. J. Chem. Phys. 1936, 4, 283-291.

(7) Zhurkov, S. N. Int. J. Fract. Mech. 1965, 1, 311-322.

(8) De Gennes, P. G. J. Chem. Phys. 1974, 60, 5030-5042.

(9) Bell, G. I. Science 1978, 200, 618-627. the absolute temperature. Because of the exponential acceleration of the dissociation rate, it is expected that forces on a picoNewton scale might noticeably decrease the lifetime of bound molecular species. ${ }^{1}$

Single-molecule force spectroscopy provides quantitative tools to study the effects of mechanical forces at a molecular level. ${ }^{4,10}$ The majority of quantitative experiments performed at a singlemolecule level focus on events (such as unbinding between ligands and receptors, unfolding in globular proteins, and conformational transitions in extended macromolecules) where the direction of applied force coincides or nearly coincides with an observable parameter that characterizes the reaction (i.e., length of molecules). ${ }^{10-12}$ A significant reduction in the lifetime of a receptor-ligand system under a pulling force is confirmed experimentally for many molecular systems. ${ }^{1,4,11}$ However, in these experiments the direction of applied forces is often limited by the methodology of pulling experiments. This methodological restriction significantly limits the scope of problems that can be studied.

It might be suggested that compressive mechanical forces applied to molecules at different directions might efficiently influence the course of reactions as well. For example, ensemblelevel studies have shown that enzyme distortions by $<0.5 \AA$ can significantly affect catalytic efficiency. ${ }^{13}$ Single-molecule measurements of enzyme lysozyme function have also indicated that enzyme activity is directly connected to the changes in enzyme mechanical properties. ${ }^{14}$ We hypothesize that a com-

(10) Hinterdorfer, P.; Dufrene, Y. F. Nat. Methods 2006, 3, 347-355.

(11) Clausen-Schaumann, H.; Seitz, M.; Krautbauer, R.; Gaub, H. E. Curr. Opin. Chem. Biol. 2000, 4, 524-530.

(12) Fisher, T. E.; Marszalek, P. E.; Fernandez, J. M. Nat. Struct. Biol. 2000, 7, 719-724.

(13) Berezin, I. V.; Klibanov, A. M.; Samokhin, G. P.; Martinek, K.; Klaus, M. Methods Enzymol. 1976, 44, 558-571.

(14) Radmacher, M.; Fritz, M.; Hansma, H. G.; Hansma, P. K. Science 1994, 265, 1577-1579. 

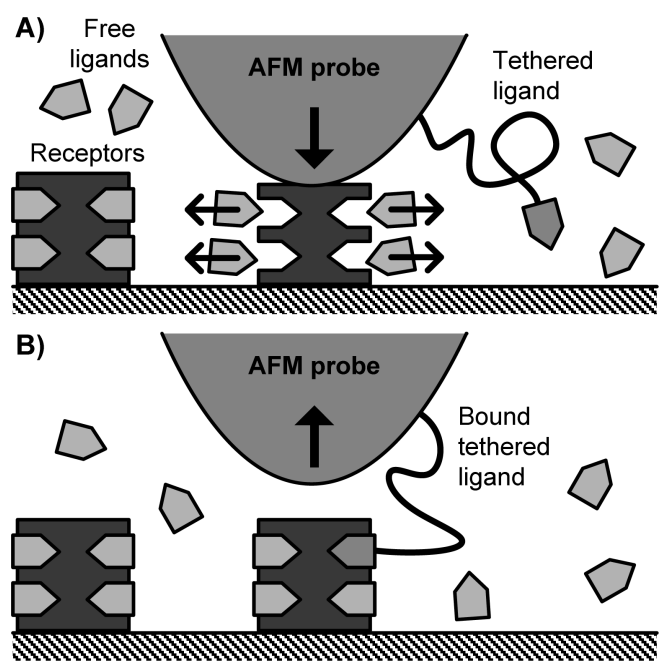

C)

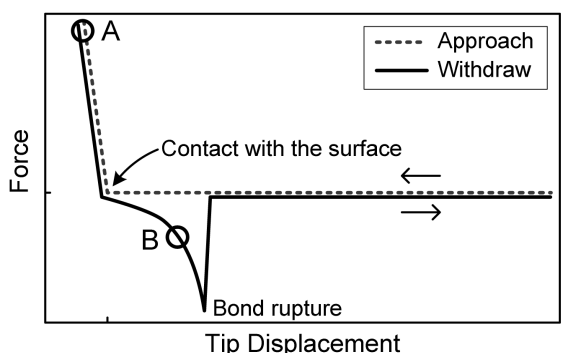

Figure 1. In the presence of a high concentration of free biotin in solution (typical concentration in our experiments is $\sim 200 \mu \mathrm{M}$, a value much higher than the dissociation constant of $\sim 10^{-15} \mathrm{M}$ ), all available sites on streptavidin molecules immobilized on a substrate are expected to be occupied by biotin molecules. (A) Distortion of streptavidin by a compressive force facilitates dissociation of biotin ligands. (B) Tethered ligand can bind to a newly vacated binding site. (C) A cartoon of the approach-withdraw cycle. Points corresponding to panels A and B are labeled on the graphs.

pressive mechanical stress applied to a receptor-ligand complex might also accelerate its bond dissociation.

Biotin-streptavidin ligand-receptor pairing provides a convenient experimental test system because of the high affinity and specificity of this interaction. ${ }^{15,16}$ To test the effects of compressive forces, experiments can be imagined that use the capability of an atomic force microscope (AFM) to apply a local force on a sample surface with the tip of a sharp probe combined with the AFM capability of detecting interaction forces between the ligands tethered to the probe and surface-immobilized receptors. ${ }^{10}$ Figure 1 shows the experimental scheme where the same AFM probe is used to apply the compressive force on the sample and to test the effects of this force on the molecular complex. The experiment proceeds by contacting the streptavidin-modified surface in the presence of free ligand with an AFM probe equipped with biotin tethered by a hydrophilic polymeric linker. If the force applied by the probe onto the bound streptavidin-biotin complex causes rapid dissociation of bound biotin, the tethered biotin ligand may bind to the newly vacated binding site. ${ }^{17}$ This binding can be detected by measuring the forces required to rupture a newly created biotin-streptavidin bond. If biotin dissociation does not occur

(15) Chilkoti, A.; Stayton, P. S. J. Am. Chem. Soc. 1995, 117, 1062210628.

(16) Merkel, R.; Nassoy, P.; Leung, A.; Ritchie, K.; Evans, E. Nature 1999, 397, 50-53.

(17) Guo, S.; Lad, N.; Ray, C.; Akhremitchev, B. B. Biophys. J. 2009, 96, 3412-3422. during the tip-sample contact, then no bond rupture events should be detected.

Competition binding experiments that are often used to test the binding specificity of a receptor-ligand pair in force spectroscopy experiments ${ }^{18-22}$ demonstrate the plausibility of this new approach. In these experiments the probability of detecting the rupture of a receptor-ligand bond is compared in solutions without a free ligand and with a high concentration of free ligand. Often it is found that the probability to detect rupture events decreases when free ligand is added to solution. However, the decreased detection probability is far from zero, even when the concentration of free ligand exceeds the dissociation constant by several orders of magnitude. Additionally, the detected bond ruptures occur at rupture forces that are similar to the rupture forces in experiments without free ligands, suggesting that the same specific ligand-receptor bonds are tested in measurements with and without free ligand in solution. In experiments where specificity of binding is tested without using competition assays, the detection probability decreases to nearly zero. ${ }^{23}$

Here we present experiments demonstrating how compressive forces exerted by an AFM tip affect the probability to detect receptor-ligand bonds that are not expected to form in equilibrium competition experiments. These measurements support a model of accelerated biotin dissociation under the AFM probe. As additional evidence we employ single-molecule "fly-fishing" experiments. ${ }^{24}$ Here the probability of the receptor-ligand binding is studied in experiments where the AFM probe stops a few nanometers above the substrate surface, thus applying no compressive force to the surface. These measurements indicate that a compressive force as low as 40 $\mathrm{pN}$ might significantly accelerate the rate of biotin dissociation. This force is smaller than the rupture force in the pulling measurements, indicating that the compressive forces might efficiently promote dissociation of molecular complexes. In addition, results reported here might be related to the role of compressive forces in the force-induced dissociation of protein molecules from protein crystals during tapping-mode imaging ${ }^{25}$ and in surface modifications by nanografting. ${ }^{26}$

\section{Materials and Methods}

1. Sample Preparation. Sample preparation procedures are similar to previously reported methods. ${ }^{18}$ A description of these procedures is included in the Supporting Information.

2. Data Collection and Analysis. All force spectroscopy experiments were conducted with an Asylum Research (Santa Barbara, CA) MFP-3D AFM in 0.1 M PBS buffer, pH 7, at $25^{\circ} \mathrm{C}$. A custom-made temperature stage was used to set the temperature, and a custom-made O-ring was used to reduce evaporation of the

(18) Guo, S.; Ray, C.; Kirkpatrick, A.; Lad, N.; Akhremitchev, B. B. Biophys. J. 2008, 95, 3964-3976.

(19) Pfister, G.; Stroh, C. M.; Perschinka, H.; Kind, M.; Knoflach, M.; Hinterdorfer, P.; Wick, G. J. Cell Sci. 2005, 118, 1587-1594.

(20) Sulchek, T. A.; Friddle, R. W.; Langry, K.; Lau, E. Y.; Albrecht, H.; Ratto, T. V.; DeNardo, S. J.; Colvin, M. E.; Noy, A. Proc. Natl. Acad. Sci. U.S.A. 2005, 102, 16638-16643.

(21) Dufrene, Y. F.; Hinterdorfer, P. Pflugers Arch. 2008, 456, 237-245.

(22) Taranta, M.; Bizzarri, A. R.; Cannistraro, S. J. Mol. Recognit. 2008, $21,63-70$.

(23) Shi, Q. M.; Chien, Y. H.; Leckband, D. J. Biol. Chem. 2008, 283, 28454-28463.

(24) Ludwig, M.; Rief, M.; Schmidt, L.; Li, H.; Oesterhelt, F.; Gautel, M.; Gaub, H. E. Appl. Phys. A-Mater. Sci. Process. 1999, 68, 173-176.

(25) Guo, S.; Akhremitchev, B. B. Langmuir 2008, 24, 880-887.

(26) Liu, M.; Amro, N. A.; Liu, G. Y. Annu. Rev. Phys. Chem. 2008, 59, 367-386. 


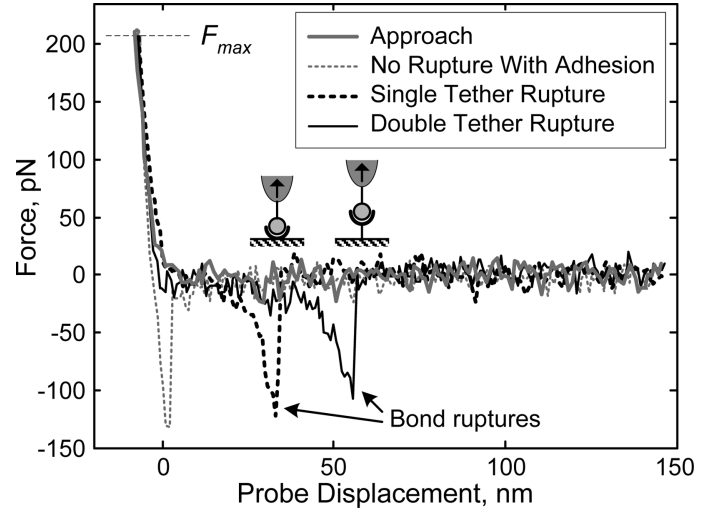

Figure 2. Typical force-displacement curves measured in presence of 200 $\mu \mathrm{M}$ of free biotin. Detected bond ruptures occur at tip-sample separations close to the length of one PEG tether in single tether experiments or two times the PEG tether length in double tether experiments. Dashed curve shows tip-surface adhesion that is sometimes detected in experiments.

phosphate buffer during the data collection. During force curve collection, the probe was raster scanned over a $5 \times 5 \mu \mathrm{m}^{2}$ square area on the substrate (force volumes with $32 \times 32$ lateral size) to obtain a good statistical average.

For free biotin concentration dependence measurements, a custom-made liquid chamber was used to exchange the biotin solutions of different concentrations on site. Measurements were conducted in the solutions with different free biotin concentrations in the range from 0 to $0.82 \mathrm{mM}$ with probe velocity of $1.5 \mu \mathrm{m} / \mathrm{s}$ and dwell time of $0.1 \mathrm{~s}$. Other parameters are described in the text above.

The force curves containing probable rupture events were detected automatically using several filters as described previously. ${ }^{18}$ These force curves were individually inspected to confirm the occurrence of rupture events. Force curves with rupture events were fit by the appropriate polymer stretching model to obtain corresponding tether contour lengths and rupture forces. Binding probability was calculated as the ratio of the force curves with rupture events to the total number of collected force curves.

In experiments where the probe was not allowed to touch the surface, no trigger settings were used. Instead the probe was extending toward the surface while gradually increasing the position of maximum extension. When contact with the surface was detected, the position of the maximum probe extension was decreased and the experiment was performed again. To estimate the minimum tip-sample separation, the detected probe contacts with the surface were used. When the probe contacted the surface, the probe displacement corresponding to the contact point was obtained. These data were used to estimate the minimum tip-sample separation when the probe did not contact the surface by linear interpolation using closest contact displacement values. Detailed description of this procedure and the corresponding data are included in the Supporting Information. This procedure compensates for noticeable vertical drift of cantilever probe position with respect to the sample surface.

\section{Results and Discussion}

1. Specificity of Measured Interactions. Typical force curves with characteristic rupture events in the presence of free biotin are shown in Figure 2. In these experiments ruptures are detected at tip-surface separations close to the length of one or two poly(ethylene glycol) (PEG) tethers. This indicates that the measured rupture events correspond to the ruptures of interactions between tethered molecules and not to the nonspecific interactions of the biotin ligand with the substrate. Using the tip-sample separation at rupture as a parameter validating the specific recognition nature of measured rupture events is similar

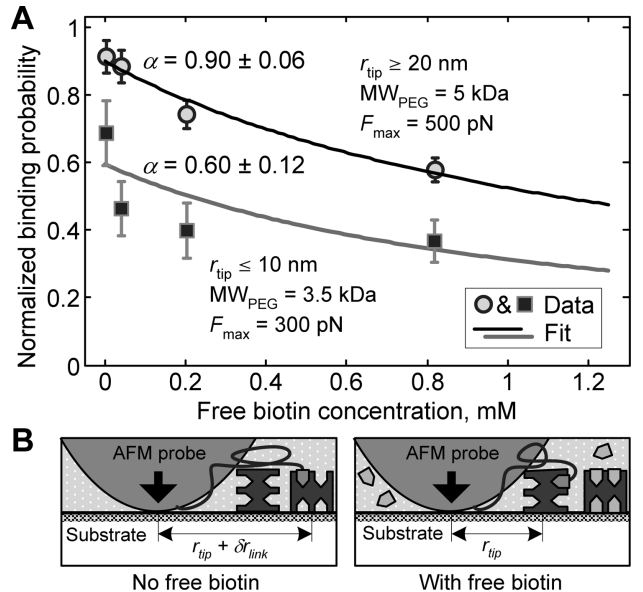

Figure 3. Competitive binding assay using two probes of different sizes. (A) Measured relative binding probability and fit by eq 1 . Results of two experiments are shown. Parameters are shown next to the graphs, including the manufacturer-supplied probe radii, length of PEG tethers, and maximum compressive force. (B) Cartoon illustrating the difference between binding probabilities for probes of different sizes. This effect is expressed by the factor $\alpha$ in eq 1 . This factor for each experiment is shown next to the graphs. On the right-hand side of this cartoon we show that the probe does not necessarily land on the streptavidin molecule directly with the apex.

to the detection of the "fingerprint" sawtooth pattern in "polyprotein" force spectroscopy measurements where distance between the adjacent rupture events is used to confirm the singlemolecule nature of the measurements. ${ }^{12}$ With only one rupture event detected in our experiments, we do not establish unambiguously that only one bond has been ruptured. However, this establishes that molecules at the end of the tether are responsible for binding (see also Figure 5D, below).

To further confirm the specific nature of measured rupture events, we have performed additional tests. In one series of tests, biotin on the tether is replaced with a tert-butyloxycarbonyl (BOC) protecting group. In our previous force spectroscopy work, ${ }^{18,27,28}$ we routinely performed such "empty tether" measurements to test whether molecules attached at the ends of tethers are responsible for ruptures. This test confirms that having biotin attached to the tether is essential for detecting ruptures. In another series of control tests, we have compared detection probability with biotin tethered to the probe before and after chemical denaturation of streptavidin immobilized on the substrate. These tests have indicated that the detection probability drastically decreases after streptavidin denaturation. Therefore, detection of rupture events requires the presence of biotin tethered to the probe and streptavidin in the native state immobilized on the substrate. Detailed description of all tests is included in Supporting Information.

2. Free Biotin Concentration Dependence. If the detected rupture events in the presence of free biotin in solution come from specific biotin-streptavidin interactions, then we expect that there will be a dependence of the probability to detect a bond rupture on concentration of free biotin. Figure 3A shows the number of detected rupture events normalized to the number of detected rupture events without free biotin in solution measured using two types of AFM probes with different radii of curvature. One probe is a silicon nitride probe with relatively

(27) Ray, C.; Brown, J. R.; Akhremitchev, B. B. J. Phys. Chem. B 2006, $110,17578-17583$

(28) Ray, C.; Brown, J. R.; Kirkpatrick, A.; Akhremitchev, B. B. J. Am. Chem. Soc. 2008, 130, 10008-10018. 
Table 1. Parameters of the Competition Binding Model (Eq 1) Derived from Results Shown in Figure $3^{a}$

\begin{tabular}{cccc}
\hline$r_{\text {tip }}, \mathrm{nm}$ & $\alpha$ & $\left(k_{\text {on }}^{\text {kink }} / k_{\text {rioe }}^{\text {tree }}\right) C_{\text {eff, }}, \mathrm{mM}$ & $R_{\mathrm{h}}, \mathrm{nm}$ \\
\hline$\sim 20$ & $0.90 \pm 0.06$ & $1.40 \pm 0.08$ & $8.3 \pm 0.2$ \\
$\sim 10$ & $0.60 \pm 0.12$ & $1.1 \pm 0.3$ & $8.9 \pm 0.8$
\end{tabular}

${ }^{a} r_{\text {tip }}$ are the approximate radii of the probes used in experiments as provided by the manufacturers and $R_{\mathrm{h}}$ are the radii of a hemisphere per one biotin obtained from $\left(k_{\mathrm{on}}^{\text {link }} / k_{\mathrm{on}}^{\text {free }}\right) C_{\text {eff }}$ parameters, assuming ${ }^{13}$ that $k_{\mathrm{on}}^{\text {link }}$ $=k_{\mathrm{on}}^{\mathrm{free}}$. The indicated errors are calculated from the experimental error and correspond to $95 \%$ confidence interval.

large radius of curvature (typically between 20 and $60 \mathrm{~nm}$ as specified by the manufacturer and confirmed by electron microscopy imaging ${ }^{29}$ ) and the full pyramidal angle of $70^{\circ}$; the second probe is a silicon probe with smaller radius of curvature $(<10 \mathrm{~nm}$ specified by the manufacturer) and the full cone angle of $30^{\circ}$. The highest concentration of free biotin is limited by biotin solubility. The lowest concentration of free biotin is 4 $\mu \mathrm{M}$, a value that by many orders of magnitude exceeds the femtomolar dissociation constant of the biotin-streptavidin recognition reaction in solution. Results in Figure 3A show that there is a competition for binding sites on streptavidin between the tethered biotin on the probe and the free biotins in solution. In competitive binding experiments, the relative probability to detect a rupture event in comparison to experiments without free biotin can be approximately calculated by

$$
P_{\mathrm{n}}=\frac{N_{\mathrm{R}}\left(C_{\text {free }}\right)}{N_{\mathrm{R}}(0)} \approx \frac{\alpha\left(k_{\mathrm{on}}^{\mathrm{link}} / k_{\mathrm{on}}^{\mathrm{free}}\right) C_{\mathrm{eff}}}{\left(k_{\mathrm{on}}^{\mathrm{link}} / k_{\mathrm{on}}^{\mathrm{free}}\right) C_{\mathrm{eff}}+C_{\text {free }}}
$$

where $P_{\mathrm{n}}$ is the relative probability to detect rupture events, $N_{\mathrm{R}}$ is a measured number of rupture events, $C_{\text {eff }}$ is an effective concentration of linked biotin, $C_{\text {free }}$ is concentration of free biotin in solution, and $k_{\mathrm{on}}^{\text {link }}$ and $k_{\mathrm{on}}^{\text {free }}$ are the on-rates for binding of linked and free biotin, respectively. $\alpha$ is a factor that accounts for the relative decrease in binding probability because of the difference in the area sampled by the linked biotin on the AFM probe: with free biotin in solution it samples only the area under the probe, but without free biotin in solution the tethered biotin can bind outside of the contact area. ${ }^{17}$ We note that eq 1 is approximate; here we use the on-rates instead of binding constants because in these experiments the measured binding probability depends on rates of association but does not depend on rate of dissociation (the lifetime of a free biotin-streptavidin bond is $\sim 50 \mathrm{~h}$, while a tethered biotin has a chance to bind to streptavidin only during $\sim 5 \mathrm{~ms}$ that the AFM probe spends within the root-mean-square (rms) tether length near the surface). Justification of using this equation is provided in the Supporting Information. The fit parameters for this model are shown in Table 1.

Factors $\alpha$ shown in Table 1 are lower than unity, consistent with the suggestion that, in competition experiments, formation of a biotin-streptavidin bond requires compressive contact of the tip with the surface. The estimated radii of a hemisphere occupied by one biotin shown in Table 1 are noticeably larger than the average rms end-to-end distance of tethers (4.5-5.5 $\mathrm{nm})$ but are still shorter than the length of fully stretched tethers $(\sim 25-40 \mathrm{~nm}){ }^{17,30}$

(29) Guo, S. L.; Akhremitchev, B. B. Biomacromolecules 2006, 7, 1630 1636.

(30) Wong, J. Y.; Kuhl, T. L.; Israelachvili, J. N.; Mullah, N.; Zalipsky, S. Science 1997, 275, 820-822.
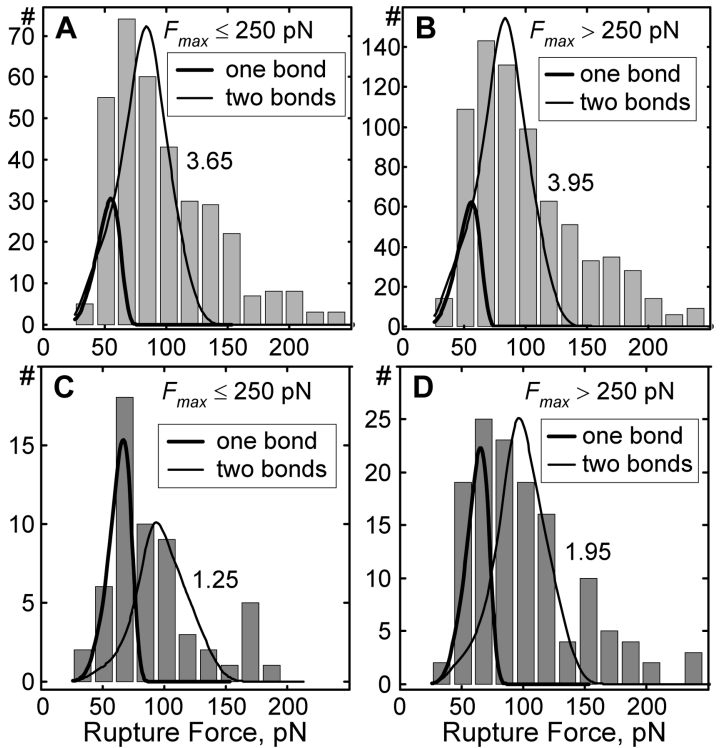

Figure 4. Histograms of rupture forces measured in solution with $200 \mu \mathrm{M}$ of free biotin. Panels A and B show histograms obtained when the probe applied force on the surface for $0.1 \mathrm{~s}$. Panels C and D show histograms obtained when the compressive force was ramped to the maximum value and then decreased without the probe resting on the surface. Limits of maximum compressive force are shown in each panel. Solid lines in these graphs were calculated using the two-bond rupture model ${ }^{14}$ and correspond to ruptures of one and two bonds as indicated in the legends. Numbers next to the two-bond rupture peaks show the ratio of the two-bond to onebond detection probabilities.

The numerical value of factor $\alpha$ depends on the relative ratio of areas and, for experiments where streptavidin is attached directly to the substrate, can be estimated by $\alpha \approx r_{\text {tip }}^{2} /\left(r_{\text {tip }}+\right.$ $\left.\delta r_{\text {link }}\right)^{2}$, where $r_{\text {tip }}$ is the radius of the tip-sample contact area where the dissociations induced by compressive force occur and $\delta r_{\text {link }}$ is the additional distance created by diffusion of the tethered ligand as illustrated in Figure 3B. Therefore, it might be expected that a decrease in the detection probability of rupture events after adding competitive ligand to solution will be more significant for probes with apex size smaller than the size of the immobilized receptor molecules. ${ }^{31}$ This suggestion is consistent with the lower value of $\alpha$ obtained for the sharper AFM probe. This model implies that the apparent efficiency of blocking of specific recognition events measured by force spectroscopy might significantly vary between measurements and does not report on the affinity of binding.

3. Distribution of Rupture Forces. The streptavidin molecule is a tetramer with four biotin binding sites. Therefore, the number of bound biotin molecules that might dissociate from a streptavidin molecule during the tip sample contact may differ. Consequently, if the number of dissociated biotin molecules is greater than 1 , then the detected rupture event might correspond to the rupture of multiple bonds and consequently occur at a higher rupture force than the rupture of a single biotin - streptavidin bond. ${ }^{18}$ This suggestion is tested by comparing distributions of rupture forces measured with maximum compression forces lower and higher than $250 \mathrm{pN}$. The resulting histograms are shown in Figure 4.

Distributions of rupture forces collected with the silicon nitride probe resting on the surface for $0.1 \mathrm{~s}$ while applying the compression force are similar for both high and low compression

(31) Averett, L. E.; Geer, C. B.; Fuierer, R. R.; Akhremitchev, B. B.; Gorkun, O. V.; Schoenfisch, M. H. Langmuir 2008, 24, 4979-4988. 

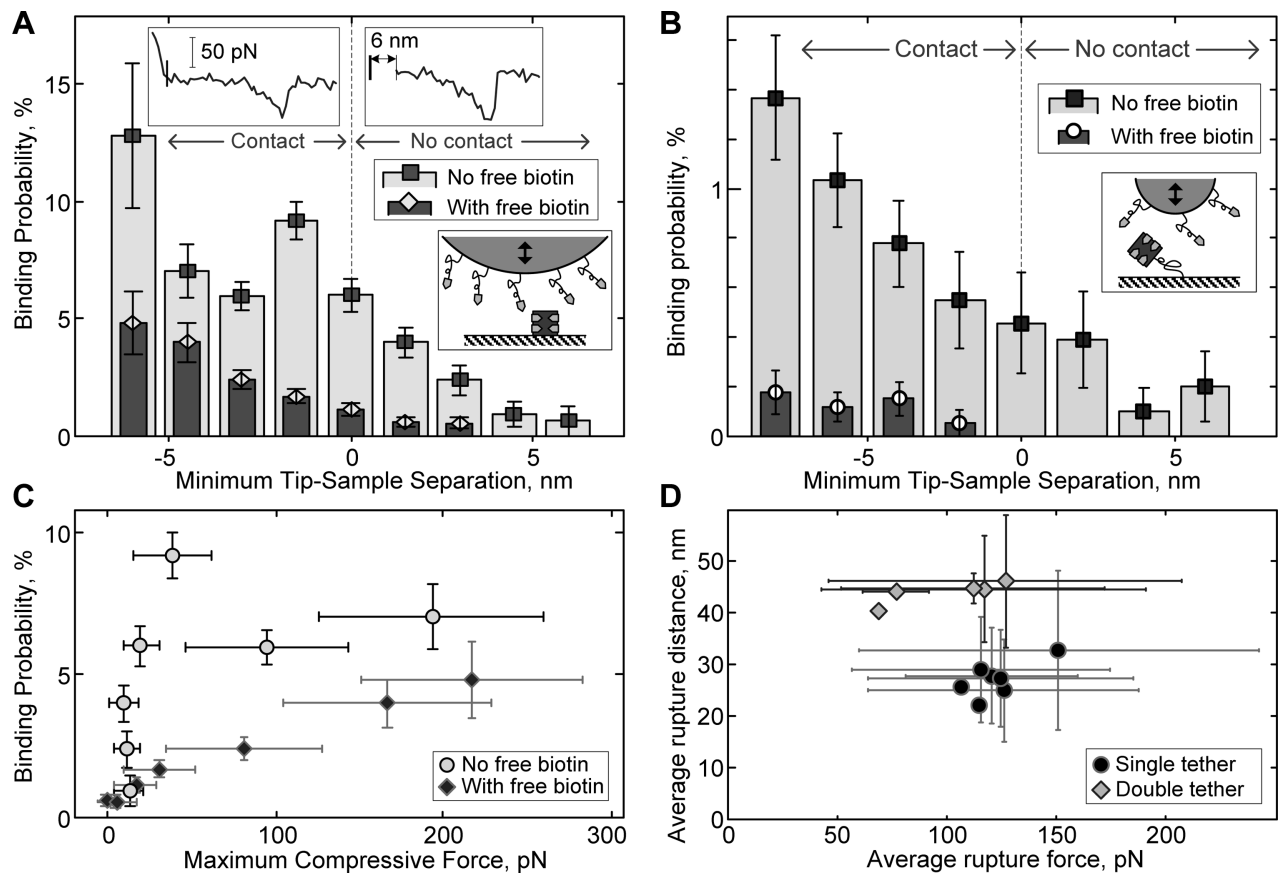

Figure 5. (A,B) Binding probability as a function of minimum tip-sample separation in single- and double-tether experiments, respectively. The insets show cartoons of these arrangements that are drawn approximately to scale. Binding probability is shown for experiments without and with free biotin in solution as indicated. The insets in panel A show two typical force plots displaying rupture events, one obtained without the tip contacting the surface and another when the tip applies compressive force onto the surface. Panel $\mathrm{C}$ shows binding probability as a function of the average maximum force applied to the sample in a single-tether experiment. Force averaging is performed over data corresponding to the histogram bins shown in panel A. Panel D shows the average tip-surface distance at rupture vs average rupture force in single- $(5000 \mathrm{Da})$ and double- $(2 \times 3500 \mathrm{Da})$ tether "fly-fishing" experiments without free biotin in solution. In all panels error bars correspond to the standard deviations.

forces (panels 4A and 4B). When the probe does not rest at the surface, the distribution of rupture forces collected with lower compression forces (panel 4C) has a noticeably higher proportion of single-bond rupture events than the distribution of rupture forces collected with higher compression forces (panel 4D). A noticeable fraction of measured rupture events occur at forces higher than the rupture force of two bonds $(\sim 150 \mathrm{pN})$ and therefore come from ruptures of more than two bonds. ${ }^{18,32}$ The proportion of these high rupture forces also increases with an increase of compression forces, as expected. It is likely that rupture forces in the range from $\sim 150$ to $\sim 220 \mathrm{pN}$ come from ruptures of three bonds, while ruptures of four bonds at forces from $\sim 220$ to $300 \mathrm{pN}$ were seldom detected. Comparison of the histograms shown in panels A and C or in panels B and D indicates that the dissociation induced by compressive forces depends on the time the force is applied to the bound molecules. Also, data shown in panels B and D indicate that there are molecular complexes capable of withstanding the application of high compressive forces, because in panel B the detection probability of rupture forces that are higher than the rupture force of a single bond is larger than in panel D.

4. "Fly Fishing" Measurements. Additional testing of the compressive force contribution to the dissociation of bound biotin was performed in experiments when the downward motion of the AFM probe was stopped prior to reaching the surface followed by a withdraw motion ("fly-fishing" mode). ${ }^{24}$ Because biotin on the probe is tethered by a flexible watersoluble linker, it might be expected that rupture events may be detectable even without the AFM probe contacting the surface

(32) Gu, C.; Kirkpatrick, A.; Ray, C.; Guo, S.; Akhremitchev, B. B. J. Phys. Chem. C 2008, 112, 5085-5092. when streptavidin binding sites are not blocked by free biotin. Experiments that use this approach are described below.

The measured dependences of binding probability on the minimum tip-sample separation are shown in Figure 5A,B for the single- and double-tether arrangements, respectively. The single-tether experiment (panel 5A) employed $5 \mathrm{kDa}$ PEG tether and silicon nitride probe; the double-tether experiment (panel 5B) employed two $3.5 \mathrm{kDa}$ PEG tethers and a sharper silicon probe (see Figure 3 for description of the probes' geometry). In both experiments effects of adding $200 \mu \mathrm{M}$ of free biotin were tested. Negative values of separation correspond to the AFM probe displacement when the tip contacts the surface-the more negative values correspond to the higher compressive forces. Histograms in panel 5A show that without free biotin there is noticeable binding probability when the tip is $\sim 6-7$ $\mathrm{nm}$ away from the surface. This probability is rapidly increasing with decreasing tip-sample separation.

After the tip touches the substrate, the binding probability does not show a systematic increase over the limited range of compressive forces corresponding to negative values of separation. In contrast, with free biotin in solution the binding probability becomes nonzero when the probe approaches the surface by distances below $3 \mathrm{~nm}$. This distance is less than the size of a streptavidin molecule $(\sim 4.5 \mathrm{~nm})$. The binding probability then gradually increases with increase of compression forces. This observation is confirmed by the dependence of binding probability on the maximum compressive force (Figure 5C) averaged over each bin of histograms from panel A. Without free biotin, a very rapid increase in binding probability occurs when compressive forces are low, corresponding to the probe either away from the surface or in the vicinity of the surface (the detected level of compressive forces 
during fast initial increase corresponds to the thermal motion of the cantilever). There is no systematic increase in binding probability with further increase in compressive force; however, it might be expected that a small dependence of detection probability on compressive force might be detected because the tip-sample contact area depends on applied force.

The specific-recognition nature of rupture forces in the double-tether measurements is supported by comparison of the tip-sample separation at rupture shown in Figure 5D. Here similar rupture forces are detected in both experiments; the tip-sample separations at rupture are consistent with the extension of a single $5 \mathrm{kDa}$ tether and two $3.5 \mathrm{kDa}$ tethers. This observation in combination with the test experiments described above supports the use of the measured rupture distance as a characteristic "fingerprint" of specific interaction similarly to using the rupture distance pattern in "polyprotein" experiments. Average rupture forces shown in Figure 5D have large standard deviations. We think that fly-fishing experiments do not discriminate against possible formation of multiple bonds because of multivalency inherent in the streptavidin receptor. ${ }^{33}$ Therefore, measured ruptures might include contributions from single and multiple bonds and thus have large standard deviations. $^{33}$

Double-tether measurements also indicate that biotinstreptavidin binding might occur when the probe-sample separation is $\sim 6 \mathrm{~nm}$ (Figure $5 \mathrm{~B}$ ). Overall binding probability is considerably lower because much fewer biotin molecules can be grafted on a shaper probe. Also, adding free biotin to solution eliminates rupture events detected without the probe contacting the surface. However, when the probe contacts the surface, the number of recovered rupture events is considerably lower than that in the single-tether fly-fishing measurements. Two explanations for this observation can be given. First, tethered streptavidin is free to move and therefore can "escape" from underneath the sharp probe. Second, immobilization of streptavidin in single-tether measurements might limit the access of biotin to some of the binding sites, and the AFM tip changes the orientation of streptavidin molecules during contact with the surface, making these sites available for binding. We have performed double-tether measurements with the silicon nitride probe that has a large radius of curvature (so that tethered streptavidin molecules cannot avoid application of compressive forces). The probability to detect rupture events with free biotin measured in these experiments is similar to or even higher than in the single-tether experiments with the same type of probe. Therefore, the second explanation is unlikely. The first explanation is also supported by results of the competition binding assay shown in Figure 3, further reinforcing our explanation of the nature of rupture events when free biotin is added to solution.

Results in Figure 5C show that with free biotin in solution, binding probability starts to increase when the detected compressive forces exceed the noise level ( $\sim 10 \mathrm{pN} \mathrm{rms}$ ) by a factor of 3 (at $\sim 40 \mathrm{pN}$ ). The binding probability then continues to increase with compressive force. We note that these compressive forces are probably an overestimate of true compressive forces responsible for biotin dissociation because it is likely that the probe not only contacts one streptavidin molecule but also might contact the substrate and/or other streptavidin molecules, as illustrated in Figure 3B. The onset in dissociation of biotin from streptavidin starts at the level of compressive force comparable

(33) Guo, S.; Li, N.; Lad, N.; Desai, S.; Akhremitchev, B. B. J. Phys. Chem. C 2010, 114, 8755-8765. to or even lower than that in pulling experiments (compare with rupture forces in Figure 4). However, the compressive force is applied directly by the probe without polymeric tethers; the tethers significantly decrease the loading rate. ${ }^{34}$ If in biotinstreptavidin pulling experiments the force were applied without using the tether, the bond rupture would occur at a pulling force of $\sim 100 \mathrm{pN} .{ }^{18}$ These observations indicate that there is an efficient coupling between the compressive force and the dissociation pathway that might include some "lever arm action". 35

5. Alternative Explanations. One additional potential explanation of the detected biotin-streptavidin bond formation after the probe makes contact with the surface might be that there is some small population (small so that the ensemble averaged association constant is still high) of streptavidin molecules that are folded imperfectly and thus cannot bind biotin but somehow become active after the contact with the AFM probe. We think that this explanation is unlikely because the sample preparation procedure used here creates low grafting densities of streptavidin $^{36}$ and the binding probabilities with and without free biotin in solution are similar and low (below 10\%). Therefore, a small sub-population of "imperfect" streptavidin molecules cannot be responsible for the observed effect. A more restricted suggestion that only one of four binding sites is "imperfect" and is "fixed" by application of compressive force is also unlikely because if this were the case the solution experiments would report a 3:1 biotin-streptavidin binding ratio, which contradicts experimental results. ${ }^{15}$

\section{Conclusions}

Here we have demonstrated that compressive forces can accelerate dissociation of the bound ligand from a receptor. This observation explains why competitive binding assays in force spectroscopy show binding constants considerably lower than those obtained in measurements by other (force-free) techniques. We expect that this to be a general rule for the competitive binding assays by AFM as currently implemented. Here, it was also observed that sometimes even relatively low compressive forces in altering dissociation kinetics might significantly increase the rate of dissociation. This surprising effectiveness of compressive forces might lend support to the suggestion that uniform pressure applied to the surface of protein can be amplified inside a protein molecule. ${ }^{37}$ However, our results also show that some biotin-streptavidin bonds can withstand the application of even large compressive forces (see Figure 4). This indicates that the direction of compressive force is important in promoting efficient dissociation. Therefore, we suggests that the technique developed here can be used to directly study effects of forces on switching protein functions. ${ }^{2,38}$ Such experiments will require carefully controlled orientation of protein molecules immobilized on substrates. Moreover, the biotin-streptavidin bond is remarkably strong, suggesting that if the observed effect can be extrapolated to weaker molecular bonds then even low pico-Newton level forces acting between molecules might be biologically important in regulating binding between biomolecules. Furthermore, effects of mechanical forces

(34) Ray, C.; Brown, J. R.; Akhremitchev, B. B. J. Phys. Chem. B 2007, 111, 1963-1974.

(35) Kraut, D. A.; Carroll, K. S.; Herschlag, D. Annu. Rev. Biochem. 2003, 72, 517-571.

(36) Hinterdorfer, P.; Gruber, H. J.; Kienberger, F.; Kada, G.; Riener, C.; Borken, C.; Schindler, H. ColloidsSurf. B-Biointerfaces 2002, 23, $115-123$.

(37) Vanselow, D. G. Biophys. J. 2002, 82, 2293-2303.

(38) Vogel, V. Anпu. Rev. Biophys. Biomol. Struct. 2006, 35, 459-488. 
on biomolecules are expected to be rather diverse. ${ }^{1,2,38,39}$ It might be expected that, for interactions that involve flexible biomacromolecules (e.g., DNA binding to proteins), the dissociation rate depends on compressive forces differently than described above. Therefore, the ubiquity and importance of the effect reported here requires further investigation.

(39) Trepat, X.; Deng, L. H.; An, S. S.; Navajas, D.; Tschumperlin, D. J.; Gerthoffer, W. T.; Butler, J. P.; Fredberg, J. J. Nature 2007, 447, 592596.
Acknowledgment. The authors thank NSF grant CHE-0719043 for financial support.

Supporting Information Available: Detailed description of the sample preparation procedures, control, and "fly fishing" experiments. This material is available free of charge via the Internet at http://pubs.acs.org.

JA1011756 